\section{Histoire Épistémologie Langage}

43-2 | 2021

Linguistique psychologique et sémiotique : le contexte allemand et son influence

\title{
La correspondance d'Émile Benveniste et Roman Jakobson (1947-1968)
}

Chloé Laplantine, Pierre-Yves Testenoire, Emile Benveniste et Roman Jakobson

\section{OpenEdition}

\section{Journals}

Édition électronique

URL : https://journals.openedition.org/hel/1284

DOI : $10.4000 /$ hel. 1284

ISSN : 1638-1580

Éditeur

Société d'histoire et d'épistémologie des sciences du langage

Édition imprimée

Date de publication : 31 décembre 2021

Pagination : 139-168

ISBN : 9791091587150

ISSN : 0750-8069

Référence électronique

Chloé Laplantine, Pierre-Yves Testenoire, Emile Benveniste et Roman Jakobson, « La correspondance d'Émile Benveniste et Roman Jakobson (1947-1968) », Histoire Épistémologie Langage [En ligne], 43-2 2021, mis en ligne le 01 décembre 2021, consulté le 17 décembre 2021. URL : http:// journals.openedition.org/hel/1284; DOI : https://doi.org/10.4000/hel.1284

HEL is licensed under a Creative Commons Attribution-NonCommercial-NoDerivatives 4.0 International License 


\section{LA CORRESPONDANCE D’ÉMILE BENVENISTE ET ROMAN JAKOBSON (1947-1968)}

\section{Chloé Laplantine ${ }^{1}$ et Pierre-Yves Testenoire ${ }^{2}$}

${ }^{1}$ CNRS, Histoire des théories linguistiques, Paris, France.

2 INSPÉ de Paris, Sorbonne Université, Histoire des théories linguistiques, Paris, France.

\begin{abstract}
Résumé - Cet article présente et édite 21 lettres issues de la correspondance entre Émile Benveniste et Roman Jakobson de 1947 à 1968. Cette correspondance fait apparaître la proximité de ces deux linguistes, qui se sont rencontrés ponctuellement, se lisaient et se tenaient informés de leur activité scientifique (qui dépasse le domaine de la linguistique puisqu'elle s'étend aux domaines de la poétique, du folklore, de la mythologie). Au-delà, ces lettres apportent une documentation sur l'histoire des structuralismes européen et américain, sur les réseaux intellectuels et la circulation des idées entre Europe et Amérique dans la période de l'après Seconde Guerre mondiale.
\end{abstract}

Mots-clés - Benveniste (Émile), Jakobson (Roman), histoire du structuralisme
Abstract - We here provide a presentation and edition of 21 letters from the correspondence of Émile Benveniste and Roman Jakobson between 1947 and 1968. This correspondence shows the closeness of the two linguists, who met occasionally, read each other's work and kept each other informed of their scientific activity (which goes beyond linguistics since it extends to the fields of poetics, folklore and mythology). Beyond that, these letters provide documentation on the history of European and American structuralism, intellectual networks, and the circulation of ideas between Europe and America in the post-World War II period.

Keywords - Benveniste (Émile), Jakobson (Roman), history of structuralism

Nous publions ici la correspondance entre Émile Benveniste et Roman Jakobson ${ }^{1}$. Après la publication des lettres entre Jakobson et Lévi-Strauss (Jakobson \& LéviStrauss 2018, complétée par Testenoire 2019) et des lettres de Benveniste à LéviStrauss (Joseph, Laplantine \& Pinault 2020), ces nouveaux documents viennent fermer le triangle des échanges entre trois acteurs majeurs du structuralisme.

La correspondance publiée se situe entre 1947 et 1968. Elle ne couvre pas l'intégralité des échanges entre les deux linguistes, leurs archives d'avant-guerre ayant dans une large partie été perdues. Il est certain que des échanges antérieurs à 1947 ont existé. Le premier point de contact avéré entre les deux savants date

1 Nous remercions Linda R. Waugh, directrice exécutive du Roman Jakobson Trust, de nous avoir autorisés à publier cette correspondance, ainsi que John Joseph et Jacqueline Léon pour leur relecture attentive de ce travail et pour leurs remarques qui nous ont permis d'éclaircir certains points. 
de $1926^{2}$. Émile Benveniste est présent à la séance de la Société de linguistique de Paris du 15 mai 1926 où Jakobson a fait le déplacement pour être présenté comme membre par Paul Boyer ${ }^{3}$ et André Mazon ${ }^{4}$. Une fois élu, Jakobson ne revient plus aux séances de la Société, mais les deux hommes se retrouvent à intervalles réguliers dans l'entre-deux-guerres, au rythme des congrès internationaux de linguistique. Benveniste n'assiste pas au premier Congrès international des linguistes de La Haye en 1928 où la contribution commune de Jakobson, Karcevski et Troubetzkoy fait connaître les positions du Cercle linguistique de Prague sur la scène internationale. Les deux savants se retrouvent au congrès suivant, à Genève en 1931, même si aucun des deux n'y fait de communication. $\mathrm{Au}$ début des années trente, Benveniste prend connaissance des deux premiers volumes des Travaux du Cercle de Prague que son directeur, Mathesius, lui envoie $^{5}$. Le second numéro est entièrement constitué de la monographie de Jakobson, Remarques sur l'évolution phonologique du russe comparée à celle des autres langues slaves. Benveniste est tenu informé des recherches phonologiques des Praguois, ne serait-ce que par les comptes rendus qui paraissent dans le Bulletin de la Société de linguistique de Paris. Parallèlement, les membres du Cercle de Prague découvrent les premiers travaux de Benveniste. Havránek signale ainsi l'importance de la publication de sa thèse, Origines de la formation des noms en indo-européen (Kobližek \& Krásová 2019 : 167).

Le Quatrième Congrès international des linguistes qui se tient à Copenhague en 1936 - année de la mort de Meillet - marque la montée en puissance d'une nouvelle génération. Benveniste et Jakobson sont tous deux chargés de lire un rapport en séance plénière. Celui de Jakobson porte « Sur la théorie des affinités phonologiques des langues", celui de Benveniste s'intitule "Problèmes de morphologie indo-européenne ». Le congrès de Copenhague est vécu par les Praguois comme le triomphe tant attendu de la méthode structurale. «À Copenhague, écrit ainsi Troubetzkoy, il est apparu pour la première fois que non seulement nous occupions des postes clés, mais encore que nous avions derrière nous des jeunes qui avaient étudié nos écrits et étaient capables de travailler de manière autonome» (Troubetzkoy 2006 : 423). Parmi ces jeunes : Benveniste

2 Françoise Bader (2012 : 232) fait l'hypothèse que Meillet, avec qui Jakobson était en contact depuis 1922 , a pu mettre en relation les deux hommes. Sans être invraisemblable, cette hypothèse n'est corroborée par aucun document.

3 Paul Boyer (1864-1949), slaviste français, premier professeur sur une chaire de russe à l'École des langues orientales en 1891, fondateur avec Antoine Meillet et André Mazon de la Revue des études slaves en 1921.

4 André Mazon (1881-1967), spécialiste de la littérature russe du XIX ${ }^{\mathrm{e}}$ siècle (on lui doit des études sur les œuvres de Dostö̈evski, Gontcharov, Pouchkine, Tolstoï, Tourgueniev) et de folklore, professeur de « Langue et littérature slaves » au Collège de France de 1923 à 1951. Il est partie prenante d'une controverse qui l'oppose notamment à Jakobson dans les années 1940 à propos de l'authenticité du poème épique connu sous le nom de Slovo d'Igor (voir la lettre du 17 mars 1948). Mazon consacre une étude importante au Slovo (Mazon 1940) où il cherche à démontrer l'inauthenticité du poème, connu par une copie datant de la fin du XVIII ${ }^{\mathrm{e}}$ siècle. Ce travail est contesté par Roman Jakobson et Henri Grégoire dans un enseignement donné à l'École libre des hautes études de 1942 à 1944 et dont le résultat sera publié en 1948 (Grégoire, Jakobson \& Szeftel 1948). Concernant cette controverse, voir Bossuat (1948 : 123-124) et Roudet (2011 : 55-67).

5 Voir la lettre de Benveniste à Mathesius du 5 janvier 1930 (Havránková \& Petkevič 2014 : 553-554). 
dont le travail sur la morphologie indo-européenne intéresse les Praguois. Il est invité, dans la foulée du congrès, à donner une conférence au Cercle linguistique de Prague. Elle a lieu le 8 mars 1937 et porte sur «l'expression linguistique de la quantité ». Le 12 mars, il donne une seconde conférence sur «la structure de l'indo-européen » à la faculté des arts de l'université Masaryk de Brno où Jakobson enseigne. Ce dernier rend compte de cette conférence dans le journal tchèque Lidové noviny. Il y souligne la cohérence de vue entre l'approche de Benveniste et celle menée par les Praguois (Jakobson 2014 [1937]).

Le mitan des années trente voit donc les liens entre les deux linguistes se resserrer. Début 1938, Benveniste est élu membre étranger du Cercle linguistique de Prague. Il participe aussi aux travaux de la Société française de phonologie qui vient de se créer sous l'impulsion de Martinet. En 1939, il contribue au volume des Travaux du Cercle de Prague en hommage à Troubetzkoy avec un article intitulé «Répartition des consonnes et phonologie du mot», un de ses rares textes en phonologie ${ }^{6}$. La même année paraît " Nature du signe linguistique » dans le premier numéro d'Acta Linguistica, la «Revue internationale de linguistique structurale » à laquelle Jakobson contribue également.

Ces convergences de plus en plus affirmées se heurtent au choc de la guerre. Jakobson fuit l'invasion nazie de la Tchécoslovaquie. Il trouve refuge à Copenhague, Oslo puis Uppsala. Au printemps 1941, il embarque à Göteborg sur un cargo à destination des États-Unis. Il passera la fin de la guerre à New York, enseignant à l'École libre des hautes études et à l'Université Columbia (voir Testenoire, à paraître). Benveniste, quant à lui, est fait prisonnier; il parvient à s'enfuir et se réfugie en Suisse. La guerre interrompt tout contact entre eux.

Ce n'est qu'après que Benveniste a retrouvé, en 1944, ses fonctions de professeur au Collège de France et à l'EPHE que les contacts scientifiques reprennent. Benveniste rend compte positivement dans le $B S L$ du premier numéro de Word, la revue du Cercle linguistique de New York que Jakobson a contribué à créer. De son côté, Jakobson est informé par André Martinet, dès 1945, de la reprise des activités à la Société de linguistique de Paris 7 . Le contact entre Benveniste et Jakobson est renoué, selon toute vraisemblance, en 1945-1946. Il est probable que Jakobson ait été l'intermédiaire de la rencontre entre Benveniste et Lévi-Strauss. La première lettre de la correspondance, datée de février 1947, porte précisément sur les démarches de ce dernier pour trouver une place universitaire en France à son retour des États-Unis.

Les 21 lettres que nous publions documentent une vingtaine d'années de l'activité scientifique des deux linguistes. On y lira la réception que chacun

6 Ce travail reflète l'importance prise par les recherches phonologiques à la fin des années trente mais il s'explique aussi par le destinataire du volume d'hommage. Interrogé sur les différents thèmes que Benveniste proposait pour sa conférence au Cercle linguistique de Prague, Troubetzkoy écrivait pourtant à Jakobson en 1937 : «Les écrits phonologiques de Benveniste sont d'habitude peu réussis. Il serait donc mieux de choisir la "structure des systèmes numéraux" ou la "question des noms abstraits" » (Troubetzkoy 2006 : 433). Jakobson cite « Répartition des consonnes et phonologie du mot» dans son étude de 1941 sur l'aphasie et le langage enfantin (Jakobson 1941).

7 Une lettre d'André Martinet à Roman Jakobson datée du 12 octobre 1945 est conservée dans les archives de Jakobson : MC 72, box 44, folder 12. 
réserve aux publications majeures de l'autre: Noms d'agent et noms d'action en indo-européen (1948) ou Problèmes de linguistique générale (1966) pour Benveniste; Kindersprache, Aphasie und allgemeine Lautgesetze (1941), l'édition de La geste du prince Igor (1948) et Preliminaries to Speech Analysis (1952) pour Jakobson. Si les deux linguistes ont des domaines de spécialité distincts, leurs centres d'intérêt se recoupent en plusieurs endroits. Ils partagent un intérêt commun pour les problèmes de linguistique générale sur lesquels la correspondance fait ressortir des points d'accord et de désaccord. Benveniste considère, par exemple, Kindersprache, Aphasie und allgemeine Lautgesetze comme « un des plus importants travaux qu'on ait vus ces dernières années sur la linguistique en général ${ }^{8}$, mais il exprime des réserves vis-à-vis de la théorie de l'information dont s'inspire Jakobson dans ses recherches phonologiques au début des années cinquante: "Il ne me semble pas, écrit-il, que la théorie de l'information permette de dessiner la configuration logique de la langue. Il nous faudra bien distinguer les problèmes du discours de ceux de la langue $»^{9}$. Cette différence d'approche apparaît dans la manière d'aborder les formes « déictiques », en tant que shifters (embrayeurs) chez Jakobson selon un modèle communicationnel (code, message, émetteur, récepteur), et en tant qu'indices de l'activité du sujet dans la langue chez Benveniste (voir Puech 1998 ; Toutain 2014). En 1956, dans son article intitulé «La nature des pronoms », rédigé en hommage à Jakobson, Benveniste cherche à faire apparaître une dimension nouvelle de la langue, « la langue comme activité manifestée dans des instances de discours » (Benveniste 1966 : 257), distincte de la langue comme « répertoire de signes et système de leurs combinaisons ». En 1957, Jakobson publie son article «Shifters, Verbal Categories and the Russian Verb $»^{10}$, mais loin d'une conception de l'activité du sujet dans sa langue, les embrayeurs y sont conçus comme des index, des signes qui renvoient au message. C'est encore dans le cadre d'une théorie de la communication que Jakobson envisage une «fonction poétique du langage ».

Autre objet de recherche qui réunit en effet les deux linguistes mais qu'ils abordent sous un angle différent : «les problèmes de la langue poétique $»^{11}$. Les lettres de Benveniste témoignent de l'attention qu'il porte aux travaux de Jakobson dans ce domaine. Il évalue avec perspicacité l'article de 1952, «Studies in Comparative Slavic Metrics ", comme une contribution majeure au champ de la métrique comparée ${ }^{12}$, et il lit ses nombreuses études de poétique, notamment son analyse du sonnet de Dante «Se vedi li occhi miei » (Jakobson \& Valesio 1966) qui consiste en une dissection structurale du poème, comparable à celle déjà

8 Voir la lettre du 07/02/1947. Benveniste souligne de nouveau l'importance de cette étude dans « Tendances récentes en linguistique générale » (Benveniste 1954).

9 Voir la lettre du 10/06/1953.

10 Cet article s'appuie, en partie, sur deux conférences données en 1950 : voir les notes de la lettre du 04/01/1951 qui indique que le problème des shifters/déictiques fait l'objet d'échanges entre les deux savants.

11 Voir la lettre du 15/07/1967.

12 Voir la lettre du 10/06/1953 
opérée sur le poème Les Chats de Baudelaire (Jakobson \& Lévi-Strauss 1962). En 1962, Lévi-Strauss et Jakobson sollicitent Benveniste pour relire leur étude du poème de Baudelaire (voir Joseph, Laplantine \& Pinault 2020) et publient ses commentaires en note de l'article. Cependant, quand Benveniste s'intéresse à son tour, en 1967, à la langue de Baudelaire, il le fait selon un autre point de vue - « Je suis, moi aussi, en ce moment occupé par les problèmes de la langue poétique mais à un point de vue un peu différent, celui de la sémiologie »-, même s'il concède que sur la question de la spécificité de la langue poétique, «quelques progrès ont été faits sur la voie de cette reconnaissance. En particulier R. Jakobson » (Benveniste 2011 : 184).

La correspondance que nous publions est transatlantique, non seulement parce que leurs auteurs s'écrivent de part et d'autre de l'océan, mais aussi parce qu'elle témoigne d'une circulation des idées linguistiques entre Europe et Amérique, d'une curiosité mutuelle et en même temps de divergences ou de la constitution de traditions différentes. Les lettres mentionnent les voyages respectifs des deux linguistes, pour se rendre à des congrès, donner des conférences ou enseigner. Benveniste est notamment invité à enseigner à Ann Arbor (Michigan) de juin à août 1950 dans le cadre du Summer Linguistic Institute organisé par la Linguistic Society of America. C'est à partir de cette expérience américaine que Benveniste conçoit des projets de conférences internationales d'une part sur la question de la signification, et d'autre part sur le thème de la langue et de la culture ${ }^{13}$. Par le biais de Claude Lévi-Strauss il est mis en relation avec la Fondation Rockefeller qui subventionnera le projet d'une conférence américano-européenne de sémantique (Jakobson en sera d'ailleurs évincé ${ }^{14}$ ) qui se tiendra à Nice en 1951. La Fondation subventionnera encore ses deux séjours d'enquêtes linguistiques en 1952 et 1953 sur la côte Pacifique Nord-Ouest. Benveniste présente d'ailleurs ce projet d'enquêtes à la Fondation Rockefeller sous l'angle de la circulation transatlantique des connaissances : « Je crois qu'il servirait le progrès des études linguistiques et anthropologiques en Europe en favorisant la connaissance des cultures amérindiennes et les échanges entre linguistes des deux continents $»^{15}$.

La dernière lettre de la correspondance date de mai 1968. Il y est question du Symposium international de sémiotique de Varsovie auquel les deux linguistes doivent se rendre ${ }^{16}$. La situation politique dans le bloc de l'Est retient finalement Jakobson d'y aller : ultime occasion manquée d'échange scientifique puisqu'en décembre 1969, Benveniste est victime d'un accident cérébral qui le laisse paralysé et aphasique.

Le linguiste français ne disparaît pas pour autant des écrits de Jakobson. Sa correspondance montre qu'il s'informe régulièrement auprès de ses interlocuteurs

13 Voir la lettre du 02/09/1950. L'intérêt pour le problème de la signification réunit les deux linguistes au début des années cinquante, Jakobson travaillant à la rédaction d'un ouvrage intitulé Sound and Meaning, dont il est question à plusieurs reprises dans la correspondance.

14 Voir la lettre du 04/01/1951.

15 Lettre de Benveniste à Edward F. D'Arms du 01/04/1952. Archives de la Rockefeller Foundation : RF, RG 1.2 , series $500 \mathrm{R}$, box 12 , folder 113 .

16 Sur le rôle de Benveniste dans ce Symposium, voir Coquet \& Fenoglio in Benveniste 2012 : 43-46. 
français de la santé de Benveniste. Il lui rend visite lors de ses passages à Paris et il intervient pour qu'il puisse rester au service de neurologie et neuropsychologie du professeur François Lhermitte à la Salpêtrière ${ }^{17}$. En 1972, se pose la question de la succession de la chaire de Benveniste au Collège de France. La candidature de Jakobson, un temps envisagée, ne peut aboutir en raison du règlement administratif du Collège qui fixe à 70 ans la limite d'âge pour les nouveaux professeurs (voir Jakobson \& Lévi-Strauss 2018: 299). Jakobson ne succède pas à Benveniste. Il ne rédige pas de nécrologie au moment de sa mort, mais il multiplie, en diverses occasions, les hommages : lors de ses conférences comme professeur invité au Collège de France en $1972^{18}$, dans son discours inaugural au premier congrès de l'Association internationale de sémiotique, qu'il intitule « Coup d'œil sur le développement de la sémiotique » en référence au "Coup d'œil sur le développement de la linguistique » de son confrère ${ }^{19}$, dans ses contributions aux deux volumes d'hommage en $1975^{20}$, ou encore dans le «Retrospect» du troisième volume des Selected Writings où Benveniste est désigné comme «one of the most clear-sighted linguists of our epoch » (Jakobson 1980 : 786).

\section{Conservation et transcription des lettres}

Les lettres reçues par Benveniste n'ayant pas été retrouvées ${ }^{21}$, les documents que nous publions proviennent tous des archives de Roman Jakobson conservées à l'Institute Archives and Special Collections of the Massachusetts Institute of Technology (Roman Jakobson Papers, MC 72). Elles sont réparties sous plusieurs $\operatorname{cotes}^{22}$. Il s'agit donc ici des lettres reçues par Jakobson et des copies des lettres qu'il a envoyées à Benveniste.

L'édition est établie d'après les manuscrits et les tapuscrits - la plupart des lettres sont, en effet, dactylographiées - conservés dans les Roman Jakobson Papers. Les conventions d'édition sont les suivantes :

*x* indique un texte barré.

17 Voir Jakobson \& Lévi-Strauss 2018 : 299-300. Dans un post-scriptum à la lettre à Lévi-Strauss du 18/07/1972 non transcrit dans l'édition d'E. Loyer et P. Maniglier, Jakobson écrit : «I am so happy to learn from Père Menasce and from Dr. Moinfar about the progress of Benveniste's reeducation and recovery. I agree with both of them that it is very important to keep him at the Salpetrière as long as possible. I am writing in this sense to Lhermitte. Please help on this question if you can » (MC 72, box 4, folder 32). Voir également les échanges avec Moinfar et Lhermite : MC 72, box 23, folders 5 et 12.

18 Voir le compte rendu qu'en fait Mitsou Ronat (1972).

19 La communication de Jakobson commence ainsi : «Émile Benveniste que je viens de voir à Paris m’a prié de transmettre au Premier Congrès de l'Association internationale de sémiotique ses souhaits de réussite, et c'est à la belle étude de notre illustre confrère "Coup d'œil sur le développement de la linguistique” (1963) que j'emprunte l'en-tête de mon exposé » (Jakobson 1979 : 3). Émile Benveniste a été le premier président de l'Association internationale de sémiotique. Jakobson lui a succédé après son accident, voir les notes de la lettre du 16/05/1968.

20 Voir Jakobson 1975a, 1975b.

21 Il n'est pas exclu qu'une partie de cette correspondance soit actuellement entre des mains privées, mais nous n'en avons pas identifié le ou les détenteurs.

22 La majorité des lettres sont conservées sous la cote MC 72, box 40, folder 4, mais trois autres figurent sous d'autres cotes : box 11, folder 28 ; box 12, folder 44 et box 138 . 
Les chevrons $<\mathrm{xxx}>$ indiquent un texte marginal, interlinéaire ou en surcharge. L'italique rend les soulignements ; l'orthographe a été lissée.

[1]

ÉMILE BENVENISTE À ROMAN JAKOBSON 07/02/1947

Roman Jakobson Papers, MC 72, box 40, folder 4

1 rue Monticelli (14 $)$

Paris, le 7 février 1947

Mon cher ami,

Je m'excuse très vivement d'avoir tant tardé à vous répondre. J'avais plusieurs raisons de ne pas vous écrire immédiatement, celle-ci d'abord que je voulais lire votre livre sur l'aphasie ${ }^{23}$. Ma lecture que divers empêchements ont ralentie, me laisse l'impression que c'est un des plus importants travaux qu'on ait vus ces dernières années sur la linguistique en général. La portée va au-delà même de son objet qui est déjà d'intérêt majeur. Je souhaite qu'il attire l'attention des psychiatres autant que des linguistes ${ }^{24}$.

Je pensais $<$, > à la suite de votre lettre $<$, $>$ que le projet dont vous parliez allait prendre corps et que je <pourrais> vous répondre avec quelque précision. Mais jusqu'ici je n'ai entendu parler de rien ${ }^{25}$. Il me reste donc simplement à vous dire que Lévi-Strauss me paraît être un des meilleurs ethnographes actuels et que sa pensée s'affirmera d'une manière très personnelle. Ce que je connais de ses travaux me fait souhaiter que son mérite soit reconnu et qu'il puisse librement poursuivre des recherches dont j'apprécie l'importance ${ }^{26}$.

Je vous écris ceci exactement à la veille de partir en mission linguistique pour $1^{\prime} \operatorname{Iran}^{27}$. J'y resterai quelques mois et espère y faire d'utile besogne. Mais à mon

23 Jakobson, Roman. 1941. Kindersprache, Aphasie und allgemeine Lautgesetze. Uppsala : Almqvist \& Wiksells.

24 En écho, l'article de Jakobson en hommage à Benveniste, « Les règles des débats grammaticaux » (titre anglais : «On Aphasic Disorders from a Linguistic Angle»), s'ouvre sur cette dédicace : « C'est à Émile Benveniste qui fut l'un des premiers à soutenir l'importance des études strictement linguistiques sur les syndromes de l'aphasie que je tiens à dédier, en hommage d'admiration et d'affection, cette étude » (Jakobson 1975a : 11).

25 Selon toute vraisemblance, il est fait allusion ici aux démarches de Claude Lévi-Strauss pour trouver un poste universitaire à Paris. Celui-ci rentre en France à l'automne 1947.

26 Concernant la relation Benveniste-Lévi-Strauss, voir Bader (2012), Loyer (2015 : 339-42 ; 353-56), Jakobson \& Lévi-Strauss (2018 : 75-76), Joseph, Laplantine \& Pinault (2020). C'est semble-t-il en 19461947 que Benveniste et Lévi-Strauss commencent à discuter autour de la thèse de Lévi-Strauss. Celui-ci soutient son doctorat d'État le 5 juin 1948 (thèse principale : Les structures élémentaires de la parenté ; thèse complémentaire : La vie familiale et sociale des Nambikwara). Émile Benveniste est dans le jury.

27 Une description de cette mission se trouve dans l'Annuaire du Collège de France (1947-1948: 147) : «M. BENVENISTE, chargé de mission par le ministère des Affaires étrangères, a accompli un voyage en Iran et en Afghanistan entre février et octobre 1947. Il s'est rendu d'abord à Téhéran où il a séjourné plusieurs semaines et donné trois conférences sur les études iraniennes, l'une à l'Académie, la seconde à l'Institut franco-iranien, la dernière à l'Université. Il a mené des enquêtes linguistiques sur trois régions différentes : à Persépolis, où il a recueilli des données sur le parler de Sivand; dans les montagnes qui s'étendent au nord de Qazvin, contrée peu explorée où il a enregistré des spécimens inédits ; enfin sur les 
vif regret les interminables démarches de toute sorte qu'entraîne aujourd'hui un pareil déplacement m'ont empêché de terminer l'article que je comptais vous envoyer pour Word ${ }^{28}$. Mais ce n'est que partie remise et dès mon retour vous l'aurez ${ }^{29}$.

Pardonnez-moi ma hâte et laissez-moi vous souhaiter plein succès dans votre nouvel enseignement ${ }^{30}$ et dans vos travaux.

Votre amicalement dévoué,

\section{E. Benveniste}

[2]

\section{ROMAN JAKOBSON À ÉMILE BENVENISTE \\ $17 / 03 / 1948$ \\ Roman Jakobson Papers, MC 72, box 40, folder 4}

Prof. Emile Benveniste

March 17, 1948

Collège de France

11 place Marcelin Berthelot, Paris $5^{\mathrm{e}}$

Dear Friend,

It is quicker when I dictate, and for this reason I write you in English.

I hope very much to be able to come to this Congress and to meet you ${ }^{31}$. There

rives de la Caspienne, à Babolsar, où il a pu compléter appréciablement nos connaissances du māzandarānī. En juin il a quitté l'Iran et, à travers le Balutchistan et le N.-O. de l'Inde, il a gagné l'Afghanistan. Pendant son séjour à Kabul, il a sur l'invitation du Ministre de l'Instruction publique et de l'Académie afghane, dressé un système de transcription des sons du pashto et du persan qui fixera l'usage officiel. Répondant à une demande des autorités, il a fourni des suggestions pour résoudre la question si difficile de la place à faire au pashto dans l'enseignement, ainsi qu'un plan sommaire pour la description de cette langue. Outre un tour à Bamiyan, il a fait deux voyages d'enquête linguistique. Le premier a été consacré à une étude du parāchi, encore peu connu, dont il a délimité l'aire et exploré les trois variétés, entre la vallée du Shutul et la province du Nijrau. Au cours du second voyage, il a parcouru pour la première fois la région qui va de Faizabad et du Badakhshan à l'Oxus, entre l'Hindukush et le Pamir. Quantité de données nouvelles ont été recueillies sur les localités et les parlers de cette contrée frontière, si difficile d'accès et qui avait été jusqu'ici interdite. On en rapporte de grands vocabulaires et des spécimens variés, notamment sur le shugni, le wakhi, le sanglechi et l'ishkashimi. »

28 Word est la revue publiée par le Linguistic Circle of New York. Le Cercle est fondé à l'automne 1943 et la revue paraît à partir de 1945. Jakobson est un des membres fondateurs du Cercle - il en est le viceprésident jusqu'en 1949 - et il est membre du comité éditorial de la revue Word.

29 Le prochain article de Benveniste dans cette revue est « La négation en Yuchi », paru en août 1950 dans Word 6(2). Il fait suite aux recherches de Benveniste sur la négation (son enseignement au Collège de France en 1937-1938) et constitue son premier article sur une langue américaine. Il ne s'agit sans doute pas de celui-là.

30 Jakobson est recruté à l'Université Columbia en 1946 à la chaire Thomas G. Masaryk d'études tchécoslovaques nouvellement créée. Il occupe cette chaire jusqu'en 1949.

31 Le Sixième Congrès international des linguistes (CIL) s'est tenu à Paris du 19 au 24 juillet 1948. Joseph Vendryes est président du comité d'organisation. Benveniste en est le vice-président aux cotés de Marcel Cohen et d'Alfred Ernout; Michel Lejeune est secrétaire général. Le congrès réunit environ six cents participants malgré la proximité de la fin de la guerre. Les délégations américaines et soviétiques sont très peu représentées, les Allemands résidant en Allemagne ne sont pas invités. Pour ce premier CIL d'aprèsguerre, et après l'annulation du congrès de Bruxelles prévu en septembre 1939, une thématique unique mais 
are so many problems I would be happy to discuss just with you. I have my leave for the Winter Session of the coming academic year during which I hope to finish the book, "Sound and Meaning ", summarizing my research in general linguistics, especially in phonemics ${ }^{32}$. I sent you our last publication, « La Geste du Prince Igor $»^{33}$. It is a philological edition with detailed commentaries especially in language problems. I hope it will be reviewed in the Bulletin, and may I suggest Tesnière $^{34}$ as the reviewer, for he is an impartial man in Gregoire's ${ }^{35}$ and my discussion with the Paris Slavists ${ }^{36}$. I am informing him simultaneously about this suggestion.

Looking forward to having some news from and about you, with best wishes from both of us,

Yours as ever,

Roman Jakobson

large est choisie : la morphologie générale. Il est organisé autour de quatre questions. Jakobson est chargé du rapport de la deuxième question.

32 Sound and Meaning est le livre de synthèse sur la phonologie auquel Jakobson travaille à partir de la fin des années 1940. Bien qu'il en reprenne le titre, ce projet ne correspond pas aux leçons données à l'École libre des hautes études en 1942 qui seront publiées en 1976 sous le titre Six leçons sur le son et le sens. Véritable arlésienne de la correspondance de Jakobson jusqu'au mitan des années cinquante, l'ouvrage ne paraîtra pas. Un important dossier consacré à ce projet, comportant des plans et des chapitres entièrement rédigés, est conservé dans les Jakobson Papers : MC 72 box 33, folders 9-25.

33 La geste du prince Igor, épopée russe du XII siècle. 1948. Texte établi, traduit et commenté sous la direction d'Henri Grégoire, Roman Jakobson et Marc Szeftel, assistés de J. A. Joffe. In- $8^{\circ}, 383$ pages, 1 carte, 11 illustrations. New York : Rausen Brothers (Annuaire de l'Institut de philologie et d'histoire orientales et slaves, 8). Cette publication est le résultat d'un séminaire interdisciplinaire que Jakobson anime avec Henri Grégoire à l'École libre des hautes études de 1942 à 1944. Robert Bossuat, dans un compte rendu de l'ouvrage paru dans la Bibliothèque de l'École des Chartes, explique que l'authenticité de cette poésie épique, qui passe pour «l'un des plus précieux vestiges de l'ancienne littérature slave » et fut connue par une copie exécutée à l'intention de Catherine II en 1795-1798, a été contestée dès le XIX'e siècle et plus récemment par André Mazon. "S'élevant contre de telles conclusions, une équipe de savants réunis par le fait des circonstances à l'École des Hautes-Études, à New York, s'est donné pour tâche de réhabiliter la Geste et d'en procurer une édition définitive avec traduction et commentaires » (Bossuat 1948 : 123-124).

34 Lucien Tesnière (1893-1954), spécialiste des langues slaves, est alors professeur à l’Université de Montpellier.

35 Henri Grégoire (1881-1964) est un byzantiniste belge. Il est un des membres fondateurs de l'École libre des hautes études et il joue un rôle important dans la recréation, au sein de l'école, de l'Institut de philologie et d'histoire orientales et slaves qui existait avant-guerre à l'Université libre de Bruxelles.

36 L'expression désigne André Mazon, dont la thèse sur l'inauthenticité de la Geste du prince Igor est contestée par Jakobson et Grégoire, et André Vaillant. Les relations de Jakobson avec les slavistes français sont notoirement mauvaises, voir Chevalier 1997. 


\section{[3] \\ ÉMILE BENVENISTE À ROMAN JAKOBSON 26/04/1948 \\ Roman Jakobson Papers, MC 72, box 11, folder 28}

1 rue Monticelli

Paris le 26 avril 1948

Paris $14^{\mathrm{e}}$

Mon cher ami,

Je vous remercie de votre récente lettre et des nouvelles que vous me donnez. J'ai bien reçu votre édition de la Geste du Prince Igor, et selon votre souhait, j'ai fait parvenir l'exemplaire à $L$. Tesnière pour qu'il rende eom compte dans le Bulletin ${ }^{37}$ de ce bel et important ouvrage. Autant que j'ai pu voir, in $<1>$ me semble que vous avez gain de cause sur tous les points.

J'espère que vous ne vous laisserez pas détourner de venir en juillet au Congrès par les craintes incompréhensibles qui paraissent hanter les Américains ${ }^{38}$. Nous ne savons pas encore sur qui nous pouvons compter de manière certaine, au moins quant à l'Amérique. Mais nous souhaitons avoir votre rapport en temps voulu pour le faire reproduire ${ }^{39}$. Par ailleurs plusieurs linguistes regrettent qu'on n'ait pas laissé aux communications leur place traditionnelle. Il aurait fallu pour satisfaire tout le monde un congrès de trois semaines au moins. Je crois que notre formule est la bonne et que ce Congrès contribuera/à éclaircir quelques principes essentiels.

J'ai passé la plus grande partie de l'année dernière à explorer un certain nombre de parlers de l'Ira $<\mathrm{n}>$ et de l'Afghanista $<\mathrm{n}>$ et m'occupe en ce moment à rédiger les résultats de cette enquête ${ }^{40}$. Par ailleurs je réfléchis aux problèmes de la structure morphologique en général, mais je ne pense pas aboutir de sitôt à

37 Bulletin de la Société de linguistique de Paris 44(2). Voir Tesnière 1948.

38 Louis Hjelmslev et Eli Fischer-Jorgensen, dans le compte rendu du Sixième Congrès international des linguistes paru dans Acta Linguistica, notent que le nombre de congressistes - plus de six cents participants - « constitue environ le double par comparaison avec les congrès précédents. Pour bien évaluer ce nombre il faut encore se rendre compte du fait que l'Europe orientale d'une part, les États-Unis d'Amérique de l'autre, n'ont été représentés que par un nombre très peu élevé de congressistes, et que, conformément à une décision prise par le CILP, les linguistes allemands résidant en Allemagne n'ont pas été invités » (Hjelmslev \& Eli Fischer-Jorgensen 1950: 56-57). Les «craintes incompréhensibles » qu'évoque Benveniste font sans doute allusion à la peur du communisme qui agite l'Amérique dans le contexte de la guerre froide. On ignore les raisons de l'absence de Jakobson à ce congrès.

39 Jakobson est chargé du rapport de la question : « Dans quelles limites et dans quelles conditions l'étude synchronique et l'étude diachronique font-elles apparaître une solidarité et une interdépendance entre la structure phonétique et la structure grammaticale d'une langue ? » (Jakobson 1949a).

40 Dans sa biographie de Benveniste - texte partiellement publié dans le volume de Dernières leçons (Benveniste 2012 : 151-174) - Georges Redard explique que la publication des résultats de ces enquêtes, « [...] au total quelque 200 pages rédigées à partir des carnets de notes et quasi prêtes à l'impression », a toujours été repoussée : [note 41] «Benveniste l'a toujours remise, espérant trouver le temps de donner forme définitive à quelques parties insuffisamment élaborées à ses yeux ; elles sont simplement signalées dans ses Recherches de dialectologie iranienne (sous presses, éd. L. Reichert, Wiesbaden), où l'on trouvera, ce qui nous dispense de le donner ici, le détail des itinéraires et des enquêtes » (p. 15 du manuscrit de Georges Redard, document consultable aux Archives du Collège de France, dans le fonds Émile Benveniste). Les Recherches de dialectologie n'ont jamais été publiées. 
un exposé cohérent ${ }^{41}$. Ces problèmes sont beaucoup moins élaborés, beaucoup plus difficiles aussi que ceux de la phonétique, où la méthode phonologique a produit de si beaux résultats.

Avec l'espoir de vous revoir bientôt et de causer de ces questions avec vous, je vous envoie, pour Mme Jakobson et vous-même, mes bien amicaux sentiments,

E. Benveniste

[4]

ROMAN JAKOBSON À ÉMILE BENVENISTE

27/06/1948

Roman Jakobson Papers, MC 72, box 40, folder 4

June 27, 1948

Dear Benveniste,

As you probably already know, for pertinent technical reasons I am not able to come to the Congress, as sad as it is for me ${ }^{42}$. But I firmly hope to meet you in the not too distant future either in Paris or in this country. Thank you for your friendly words about our Igor publication and for inviting Tesnière as reviewer. This whole Summer and the Winter semester, which for me is free of lecturing, I hope to devote essentially to work on my book, " Sound and Meaning ». Maybe I will succeed in coming for a short trip to Europe, and first to Paris, sometime during the Winter session but it is not sure. In any case, let us stay in close scholarly contact. I will be happy to know what are your current literary plans.

With best wishes for the success of the Congress and for resolving the intricate morphological problems,

Yours as ever,

Roman Jakobson

41 La morphologie générale est précisément le thème unique choisi pour le Sixième Congrès international des linguistes qui se tient à Paris, comme l'indique Vendryes dans son discours d'ouverture : « Nous avons jugé plus utile de concentrer l'effort du Congrès sur l'étude d'une question unique, en la choisissant assez importante pour qu'elle puisse intéresser tous les linguistes, et assez large pour que chacun d'eux puisse y prêter une collaboration efficace du fait de sa spécialité. [...] Tel[le] est [celle] de la morphologie générale, que nous avons mis[e] à notre programme, réparti[e] en quatre questions » (Lejeune 1949 : 45). L'automne suivant, Benveniste choisit de consacrer ses cours du lundi au Collège de France à « la morphologie du verbe indo-européen, en donnant à ce terme de morphologie une acception plus étendue où les formes ne se séparent pas des emplois » (Annuaire du Collège de France 1948-1949 : 158). Après un cours sur les «problèmes de syntaxe générale » en 1949-1950, Benveniste revient aux «problèmes de morphologie générale » en 1951-1952.

42 Le rapport de Jakobson sera lu en plénière par John Lotz. Les actes du congrès indiquent que Jakobson « was not able to attend the Congress 》. 


\section{[5] \\ ROMAN JAKOBSON À ÉMILE BENVENISTE \\ $13 / 06 / 1949$ \\ Roman Jakobson Papers, MC 72, box 40, folder 4}

June 13, 1949

Dear Benveniste,

Thanks for your new book ${ }^{43}$. It is indeed remarkable for its brilliancy of method, for the elegance of presentation, and for the richness of new, concrete results. I am looking forward impatiently to your farther achievements. Such a pity that my motor accident postponed for one year my trip to Europe ${ }^{44}$. It would be so good to see you after the many years of distance.

I am awaiting with great interest the new review you have planned with LéviStrauss $^{45}$. From September my permanent address will be: Dept. of Slavic Languages, Harvard University, Cambridge, Mass ${ }^{46}$.

Most cordially yours,

Roman Jakobson

[6]

ÉMILE BENVENISTE À ROMAN JAKOBSON

06/05/1949

Roman Jakobson Papers, MC 72, box 40, folder 4

1 rue Monticelli

Paris le 6 mai 1950

Paris $14^{\mathrm{e}}$

Mon cher ami,

C'est une vraie malchance et pour moi une déception que je doive partir pour les États-Unis au moment où vous arrivez en Europe. Une des raisons qui

43 Il s'agit probablement de Noms d'agent et noms d'action en indo-européen (Benveniste 1948).

44 Jakobson est victime d'un grave accident de la route en décembre 1948.

45 Il s'agit de la revue L'Homme, fondée par Émile Benveniste, Claude Lévi-Strauss et Pierre Gourou. On trouve trace de ce projet de revue dès 1949. Le premier numéro ne paraîtra qu'en 1961. C'est dans L'Homme que paraît en 1962 l'article de Jakobson et Lévi-Strauss sur Les Chats de Baudelaire. Voir Joseph, Laplantine \& Pinault (2020).

46 En 1949, Jakobson est nommé à Harvard où il occupe la chaire Samuel Hazzard-Cross. E. Loyer et P. Maniglier indiquent dans leur édition de la correspondance Jakobson-Lévi-Strauss : « Roman Jakobson arrive à Cambridge, dans le Massachussetts, en 1949, pour occuper la chaire Samuel Hazzard-Cross de langues et littératures slaves et linguistique générale. Il semble que Jakobson ait été contraint de démissionner de Columbia dans un climat délétère de maccarthysme et de guerre froide, subissant la suspicion habituelle qui touche alors les exilés d'Europe orientale placée sous tutelle communiste » (Jakobson et Lévi-Strauss 2018 : 109). Professeur à Harvard jusqu'en 1965, émérite ensuite, Jakobson est également à partir de 1957 professeur associé au Massachussetts Institute of Technology. 
m'avaient déterminé à accepter l'invitation d'Ann Arbor ${ }^{47}$ était l'espoir de vous revoir et de reprendre nos longues conversations d'autrefois. Je voulais en particulier vous dire l'intérêt que je prends aux procédés d'analyse du phonème dont vous donnez une idée dans vos deux articles de Word ${ }^{48}$ et des travaux de Copenhague ${ }^{49}$. Heureusement je viens d'apprendre de M. Lévi-Strauss que vous comptez être de retour en Amérique en août. Tout espoir n'est donc pas perdu. Je m'embarque le 30 mai et, après quelques jours à New York, me rendrai directement à Ann Arbor. Mon enseignement m'y retiendra jusqu'au milieu d'août, après quoi mes projets sont encore mal définis. Mais avant de reprendre, dans les premiers jours de septembre, mon bateau, j'irais volontiers vous voir à Cambridge. Dites-moi si vos plans vous permettront de nous y rencontrer.

Vous trouverez ici beaucoup d'auditeurs qui connaissent depuis longtemps votre nom et vos travaux. Je ferai annoncer par la Société de linguistique vos deux conférences $^{50}$. Mais je regrette de ne pas pouvoir vous souhaiter la bienvenue ici et vous accueillir chez moi. Dites-moi au moins s'il y a quelque chose que je puisse faire pour vos conférences. Vous pourriez compter sur tous nos amis que j'avertis de votre passage ici.

Si le texte de votre conférence sur les sons et le sens n'est pas promis ailleurs, voudriez-vous nous le donner pour le Bulletin? Je souhaiterais vivement publier un article de vous ${ }^{51}$.

Dans l'espoir de vous revoir bientôt, je vous assure, mon cher ami, de mes fidèles amitiés,

E. Benveniste

47 Émile Benveniste est invité à donner un enseignement au Linguistic Institute organisé par la Linguistic Society of America à Ann Arbor (Michigan, États-Unis) de juin à août 1950. Il y occupe le temps de cet enseignement temporaire la chaire Hermann Collitz de grammaire comparée indo-européenne.

48 Il s'agit probablement des articles suivants : Jakobson, Roman \& John Lotz. 1949. Notes on the French Phonemic Pattern. Word 5(2) : 151-158 et Jakobson, Roman, Gladys A. Reichard \& Elizabeth Werth. 1949. Language and Synesthesia. Word 5(2) : 224-233.

49 Jakobson, Roman. 1949. On the Identification of Phonemic Entities. Travaux du Cercle linguistique de Copenhague 5 : 205-213.

50 Claude Lévi-Strauss est sous-directeur du Musée de l'Homme de mars 1949 à fin 1950. À son invitation, Jakobson y donne deux conférences les 20 et 23 juin 1950, la première intitulée « Les sons et les sens dans la linguistique actuelle », la seconde « La mythologie slave ».

51 Bien que membre de la Société de linguistique de Paris depuis 1926, Roman Jakobson n'a jamais publié dans son Bulletin. 


\section{[7] \\ ROMAN JAKOBSON À ÉMILE BENVENISTE \\ $18 / 07 / 1950$ \\ Roman Jakobson Papers, MC 72, box 40, folder 4}

Professor M. E. Benveniste

The Summer Linguistic Institute

University of Michigan

20 A Prescott Street

Ann Arbor, Michigan

July 18,1950

Mon cher ami :

I just came back from a most fascinating trip through seven European countries where I met so many colleagues and friends and had indeed most stimulating discussions ${ }^{52}$. Almost the whole month was spent in Paris and it is superfluous to add that I missed you at every step. Because the whole sojourn in Europe was very intense I feel a little tired now and I am not sure whether I will be able to visit the Linguistic Conference in Ann Arbor, but I am looking forward to seeing you after your sojourn in Ann Arbor either as our guest in Cambridge or if you prefer, a more restful meeting place where we could spend together relaxing days in some New England spot at the sea coast. In any case there are so many urgent problems to discuss. Please let me know your schedule and suggestions.

I hope you have a good time in Ann Arbor. Please give my best regards to our common friends.

Yours as ever,

Roman Jakobson

[8]

ÉMILE BENVENISTE À ROMAN JAKOBSON

03/08/1950

Roman Jakobson Papers, MC 72, box 40, folder 4

Michigan Union, Ann Arbor

Le 3 août 1950

Cher ami,

J'ai été enchanté d'apprendre que vous étiez rentré d'Europe et que vous en rapportiez de bonnes impressions. Vous savez combien j'ai regretté de ne pouvoir vous entendre à Paris. J'espère d'autant plus pouvoir m'entretenir avec vous dans

52 Au cours de ce voyage, Jakobson séjourne du 4 au 20 mai en Grande-Bretagne (Jakobson \& Lévi-Strauss 2018 : 127 et 134). Il y donne plusieurs conférences, dont l'une, le 10 mai, à l'Université d'Oxford dans le cadre des Ilchester Lectures, sur les vers des épopées slaves, qui sera publiée en 1952 (voir les notes de la lettre du 10/06/1953). Jakobson se rend ensuite en Scandinavie : il séjourne à Oslo et à Copenhague où il donne deux conférences, le 23 mai « Slavic Mythology » et le 25 mai « The New Doctrine of Language in Present-Day Russia » (Bank Jensen \& D’Ottavi 2020 : 143-144). À Paris, il s'exprime également à deux reprises au Musée de l'Homme les 20 et 23 juin, puis il passe deux jours à Genève où il donne une conférence sur les «Catégories verbales » devant la Société genevoise de linguistique le 29 juin (voir les notes de la lettre du 04/01/1951). 
quelques semaines. Mes plans ne sont pas enco $<$ re $>$ fixés. Après le 20 de ce mois, je pense quitter Ann Arbor et passer quelques jours à Chicago, puis me reposer un peu dans l'Est avant de regagner New York et m'embarquer le 10 sept. Il est donc probable que je pourrai vous reje rejoindre vers le $1^{\text {er }}$ sept. et passer avec vous trois ou quatre jours soit à Cambridge soit XXX eennaissez $<$ dans $>$ une localité tranquille et facilement accessible au bord de la mer, si vous en connaissez une. Je vous ferai part de mes projets avant mon départ avec plus de précision.

Ici les semaines ont passé avec une rapidité telle que toutes mes impressions se confondent en une seule expérience extrêmement agréable et instructive. J'ai les meilleurs rapports avec mes collègues et, malgré mon anglais insuffisant, mon enseignement ne marche pas trop mal. Nous avons eu en outre une série de Forum Lectures ${ }^{53}$ et la réunion de la Linguistic Society, avec une foule de problèmes et d'idées jetées dans le débat. J'aimerais beaucoup en parler avec vous, ainsi que de maintes autres choses.

Donc à bientôt. Informez-moi de vos propres intentions. J'ai grand besoin de repos après cette tension continue et ce travail dans un été subtropical, et ce sera un grand plaisir de vous revoir.

Je devrai en tout cas m'arrêter à Cambridge où Whatmough ${ }^{54} \mathrm{~m}$ 'a invité. Je l'ai plus ou moins informé de mes plans.

Amicalement à vous,

E. Benveniste

[9]

\section{ÉMILE BENVENISTE À ROMAN JAKOBSON $19 /[08] / 1950^{55}$ \\ Roman Jakobson Papers, MC 72, box 40, folder 4}

Ann Arbor, le 19/[8]/50

Cher ami,

Je viens de terminer mon enseignement et quitte demain Ann Arbor pour Chicago où je dois passer 3 ou 4 jours. Je prendrai ensuite la route de New York, probablement avec un arrêt à Buffalo. Il faut de toute manière que j'aille à Boston. Mais je serai heureux de passer auparavant quelques jours avec vous à Hunter ${ }^{56}$. Je prévois que j'arriverai pour Hudson le 25 ou le 26 août. Vous serez averti du jour et de l'heure par télégramme. Je serai heureux de vous revoir et de me

53 Benveniste donne notamment une conférence le 28 juillet 1950 intitulée « Transitive and passive ». Pour l'ensemble des intitulés des présentations, voir le Bulletin 24 de la LSA (Linguistic Society of America $1951: 4)$.

54 Joshua Whatmough (1897-1964) est alors professeur de philologie comparée à Harvard. Il a travaillé notamment sur les dialectes de la Gaule. Il a présidé le Neuvième Congrès international des linguistes à Cambridge, Mass. en 1962.

55 Nous corrigeons ici la date. Benveniste avait indiqué par erreur « 19/7/50».

56 Hunter (État de New York) est une petite ville dans les montagnes Catskill située à une quarantaine de kilomètres de la ville de Hudson. 
détendre un peu après la fatigue de cet été.

Tout amicalement votre

E. Benveniste

\author{
[10] \\ ÉMILE BENVENISTE À ROMAN JAKOBSON \\ $02 / 09 / 1950$ \\ Roman Jakobson Papers, MC 72, box 40, folder 4
}

The Biltmore

Madison avenue at $43^{\text {rd }}$ Street

New York City <cette adresse est valable jusqu'au 10 septembre>

New York, le 2 sept. 50

Mon cher ami,

Depuis que j'ai quitté Hunter, nous avons eu par Jones ${ }^{57}$ quelques nouvelles l'un de l'autre. L'atmosphère si particulière de Harvard est douce à un Européen, et ce doit être exaltant d'avoir à portée de la main tant de ressources en hommes et en instruments. Les sympathiques garçons qui travaillent avec vous m'ont fait visiter le M.I.T. (la promenade s'est achevée devant d'appétissantes langoustes), et j'ai eu plaisir à connaître Bekesy ${ }^{58}$, un de ces rares hommes de science capables de vues générales. Mais tout cela a été si rapide, et le temps me presse si fort !

Voici déjà, pour une semaine qui sera la dernière, New York et ce ciel d'orage qui est une anticipation d'Europe.

J'ai revu les Martinet avec qui nous avons parlé de vous. Ils regrettent de ne plus vous voir ${ }^{59}$.

J'ai repensé à la question dont je compte entretenir la Rock. Fondation si j'arrive à joindre $\mathrm{D}^{\prime} \mathrm{Arms}^{60}$. Il me semble maintenant qu'une rencontre internationale pour étudier le problème de la «signification» serait prématurée. Le problème n'est pas mûr ${ }^{61}$. Plus fructueuse serait une discussion du thème

57 Il s'agit probablement de Lawrence G. Jones avec qui Jakobson signera l'ouvrage Shakespeare 's Verbal Art in "Th 'Expense of Spirit" en 1970, et qui écrit avec Morris Halle un ouvrage intitulé The Sound Pattern of Russian, en 1959.

58 Georg von Bekesy (1899-1972), biophysicien d'origine hongroise. Prix Nobel de médecine en 1961.

59 André Martinet est professeur de linguistique générale et comparée à l'Université Columbia depuis 1947. Martinet écrit que sa nomination à ce poste est « a result of Jakobson intrigue » (Martinet 1994 : 28). Les deux hommes sont donc collègues à Columbia jusqu'au départ de Jakobson pour Harvard en 1949. C'est de cet éloignement dont il est question ici. Les deux savants restent cependant en contact régulier au sujet de Word dont Martinet dirige la publication, jusqu'à une brouille définitive en 1955.

60 Edward F. D'Arms, anciennement professeur de lettres classiques, est Assistant Director puis Associate Director du département des Humanités de la Rockefeller Foundation de 1947 à 1957, après quoi il travaille pour la Ford Foundation. Il est l'intermédiaire de Benveniste dans ses demandes de subventions successives: Conférence européenne de sémantique, puis ses deux séjours d'enquêtes sur la côte Nord-Ouest américaine.

61 Benveniste prend contact avec la Fondation Rockefeller dès septembre 1950 et fournit un projet : «A proposal for a discussion on the problems of meaning ». Avec les crédits de la Fondation Rockefeller, Benveniste organisera la Conférence de sémantique qui aura lieu à Nice du 26 au 31 mars 1951. Benveniste 
général «Langue et culture » qui provoque en ce moment de vives controverses (dans 1' l'American Anthropologist par exemple ${ }^{62}$ ). Qu'en pensez-vous ${ }^{63}$ ?

Ça a été pour moi une joie de vous revoir et de passer avec vous ces quelques jours qui ont été, grâce à vous deux, une fête de l'esprit et du cœur. Ce souvenir ne me quitte pas. Pour tout ce que vous avez fait, je veux vous dire, à Mme Jakobson et à vous-même, mon très affectueux remerciement.

E. Benveniste

\author{
[11] \\ ÉMILE BENVENISTE À ROMAN JAKOBSON \\ $04 / 01 / 1951$ \\ Roman Jakobson Papers, MC 72, box 40, folder 4
}

Hotel Wellington

Seventh Avenue

At Fifty-Fifth Street

New York, 19, N.Y.

New York, le 4/1/51

Cher ami,

Je suis venu pour une courte semaine, à la suite d'un arrangement improvisé à la dernière minute avec la Fondation Rockefeller, pour prendre contact avec ceux des linguistes américains qui doivent se réunir cette semaine $\mathrm{ici}^{64}$. Je regrette vivement de n'avoir aucune possibilité de voyager ni par conséquent de vous

avait l'idée d'une discussion des points de vue sur la question de la signification à laquelle des linguistes américains et européens auraient pris part. Cette discussion ne sera finalement presque qu'européenne. Elle réunit C. E. Bazell, E. Benveniste, G. Devoto, J. R. Firth, H. Frei, L. Hjelmslev, A. Sommerfelt, S. Ullmann et John Lotz (mentionné plus haut), seul participant américain.

62 Benveniste fait référence à une discussion par textes interposés qui occupe les tribunes du journal American Anthropologist [AA] en 1950. Le point de départ est un texte de C. F. Voegelin, « Linguistics without Meaning and Culture without Words » paru en 1949 dans Word (Voegelin $1949: 36-45$ ) et un texte d'I. Silva-Fuenzalida, "Ethnolinguistics and the Study of Culture » paru en 1949 dans $A A$ (SilvaFuenzalida 1949 : 446-456). C. Hockett répond à ces deux textes par une critique brève et vive : « Language “and" Culture: A Protest » publié en 1950 dans $A A$ 52(1) (Hockett 1950 : 113). Se succèdent alors les textes suivants, tous publiés en 1950 dans $A A$ 52(2) : James O. Buswell, III, « Some Comments on Hockett's "Language 'and' Culture: A Protest”" (Buswell 1950: 284-286) et Thomas A. Sebeok, «Reply to Hockett» (Sebeok 1950 : 286). On peut mentionner l'article de G. Reichard, « Language and Cultural Pattern », publié également dans $A A$ 52(2), même s'il n'est pas impliqué directement dans le débat (Reichard 1950 : 194-204), ainsi que C. F. Voegelin, "A "Testing Frame" for Language and Culture », $A A$ 52(3) (Voegelin 1950 : 432-435) et Douglas MacRae Taylor, "Language "and" or "in" Culture », $A A$ 52(4) (MacRae Taylor $1950: 559-560)$.

63 Cette discussion trouve un écho dans la Conference of Anthropologists and Linguists, qui se tient à Bloomington (Indiana) en juillet 1952. On notera l'intitulé de certaines sessions de ce congrès : « Language and Culture » et « Ethnography without Linguistics and Linguistics without Meaning ». Les résultats de ce congrès sont publiés en 1953 dans un volume intitulé Results of the Conference of Anthropologists and Linguists composé de trois chapitres rédigés respectivement par C. Lévi-Strauss, R. Jakobson, ainsi que C. F. Voegelin et T. A. Sebeok, ce dernier chapitre se référant aux échanges publiés en 1950 dans $A A$ (voir Jakobson, Lévi-Strauss, Voegelin \& Sebeok 1953).

64 Benveniste se rend à New York dans les premiers jours de janvier 1951. Le groupe de chercheurs américains est constitué de C. Hockett, F. Lounsbury, J. Lotz, R. Wells et de C. Gilpatric (Assistant Director à la Fondation Rockefeller). 
rencontrer. Vous êtes celui dont les avis, pour une étude de la sémantique, devraient être entendus en premier.

En fait, la Fondation a invité 3 ou 4 linguistes, les plus proches géographiquement, à élaborer un programme de travail. Rien de concret n'a encore été fait. Quand les véritables problèmes seront abordés, on procèdera, m'a dit Gilpatrick ${ }^{65}$, à une consultation plus large et votre participation sera requise. J'ai l'impression que la prochaine réunion sera surtout un échange de vues très générales sans aucune discussion de fond.

J'ai préféré venir maintenant, parce qu'on ne sait pas si les voyages seront encore possibles dans l'avenir ${ }^{66}$, et pour élaborer en connaissance de cause le programme de la réunion européenne, dont j'ai la responsabilité, alors que je n'en ai aucune dans l'organisation de la réunion américaine ${ }^{67}$. J'espère pouvoir réunir les 4 ou 5 participants européens à Pâques ${ }^{68}$.

Je ne sais pas exactement ce qui s'est passé à l'Unesco. J'ai appris à $24 \mathrm{~h}$ d'intervalle qu'une discussion internationale devait avoir lieu le 8 janvier, puis qu'elle était annulée. Je présume que les convocations ont été envoyées trop tard. On m'a dit d'une manière assez vague que le projet était renvoyé au mois de mai ${ }^{69}$.

Je n'ai pas besoin de vous rappeler combien la Soc. de linguistique serait heureuse de publier l'article dont vous m'avez parlé sur la structure du verbe ${ }^{70}$, et

65 Chadbourne Gilpatric est Assistant Director for the Humanities de la Fondation Rockefeller.

66 Étant donné la situation de guerre froide, J. Kuryłowicz ne pourra pas se rendre à Nice pour la Conférence de sémantique.

67 Il n'est pas certain qu'une réunion américaine ait jamais eu lieu. Jakobson est tenu à l'écart de ces discussions par ses collègues américains et par la fondation Rockefeller. Martinet s'en fait l'écho dans une lettre à Jakobson du 22 juin 1951 : « Ai vu assez longuement Benveniste qui déplore que vous et moi soyons pratiquement exclus du groupe d'études sémantiques. Il me paraît clair que János [Lotz] n'a rien fait pour que nous soyons convoqués aux parlotes organisées par Gilpatric, et Hockett et Cie freinent de toutes leurs forces » (MC 72, box 44, folder 12). Dans la correspondance interne de la Fondation Rockefeller on trouve effectivement des indices de défiance vis-à-vis de Jakobson : « [Benveniste] hoped that Jakobson would be included in the group for the Christmas meeting and said he thought he could prevent J from trying to take over the running of the meeting » (interview 19/10/1950, archives de la Fondation Rockefeller, RF, RG 1.2, series 500R, box 12, folder 112). Il n'est ensuite plus jamais fait mention de Jakobson qui n'a vraisemblablement pas été invité aux discussions. L'hostilité à laquelle est en butte Jakobson dans le champ académique américain est un fait bien documenté (voir Dixon 2007).

68 Comme indiqué plus haut, la conférence de sémantique se tient à Nice du 26 au 31 mars 1951.

69 Benveniste fait peut-être allusion à son implication dans le projet de « français élémentaire » (sur le modèle du Basic English) qui avait été lancé sous l'impulsion d'Aurélien Sauvageot, ce dernier ayant été invité en 1947 à l'Unesco à participer à une conférence sur la diffusion des grandes langues de communication. Le Centre de recherche et d'étude pour la diffusion du français (CREDIF) est créé en 1959, Benveniste fait partie du comité de suivi du projet. Pour le détail, voir Coste 2006.

70 Jakobson avait présenté une communication à la Société genevoise de linguistique le 29 juin 1950 intitulée Les catégories verbales dont les Cahiers Ferdinand de Saussure donnent le résumé suivant : "L'analyse des différences entre un terme ordinaire comme "chien" et certains termes comme "moi" - que les enfants apprennent très tard à employer et dont les aphasiques perdent très tôt l'usage - révèle dans ces derniers mots un trait particulier, un renvoi à l'énoncé dont ils font partie. Appliquée aux 3 éléments constitutifs d'une forme verbale : les participants au procès, le procès, les rapports entre participants et procès, cette distinction permet d'établir une division en 6 classes, chacune utilisant des moyens formels différents, qui englobe toute la conjugaison. Illustration de la théorie par des exemples tirés de la conjugaison russe » (Cahiers Ferdinand de Saussure $9: 6$ ). Cette communication deviendra la première partie de l'article « Shifters, Verbal Categories, and the Russian Verb » publié sept ans plus tard (Jakobson 1957). On peut supposer qu'il est question ici de ce travail en cours de préparation. 
aussi que votre petit livre sur le son et le sens, si vous pouviez en achever la rédaction, viendrait à point, au milieu des discussions sur la sémantique qui se préparent ici et en Europe.

Croyez à mes souhaits bien amicaux,

E. Benveniste

ÉMILE BENVENISTE À ROMAN JAKOBSON

22/03/1951

Roman Jakobson Papers, MC 72, box 40, folder 4

Paris, le 22 mars 1951

Cher ami,

J'ai reçu et je viens de lire avec le plus vif intérêt votre important article sur la mythologie slave ${ }^{71}$. Je souhaite que vous puissiez développer en détail ces vues nouvelles et de grande portée historique. En attendant je suis heureux de pouvoir me référer à cette notice et à l'occasion de pouvoir la citer.

Je n'ai que le temps de vous en remercier. Je pars dans une heure pour Nice où nous devons tenir la semaine prochaine une réunion de linguistes européens sur les problèmes de la «sémantique ». J. Lotz ${ }^{72}$ y assistera en qualité d'observateur américain.

Votre amicalement dévoué,

E. Benveniste

[13]

ÉMILE BENVENISTE À ROMAN JAKOBSON

$13 / 06 / 1952$

Roman Jakobson Papers, MC 72, box 12, folder 44

À MM. JaKoBSOn, FANT ET HALlE

Paris le 13 juin 1952

Tous mes remerciements pour les Preliminaries to Speech Analysis ${ }^{73}$ que vous

71 Il s'agit d'un bref article intitulé «Slavic mythology » et publié dans un dictionnaire de folklore (voir Jakobson $1950: 1025-1028)$. Calvert Watkins (1983:39-46) note que ce texte bref définissait un champ d'étude nouveau et posait les bases des réflexions futures.

72 John (ou János) Lotz (1913-1973) est un linguiste américano-hongrois, alors professeur de linguistique à l'Université Columbia. Jakobson a rencontré Lotz lors de son séjour en Suède en 1941. Leur collaboration a repris lors de l'arrivée de Lotz à New York en 1947. Jakobson et Lotz ont publié trois travaux en commun : Jakobson \& Lotz 1941, 1949, 1968.

73 Voir Jakobson, Fant \& Halle 1963 [1952]. Gunnar Fant (1919-2009) et Morris Halle (1923-2018) travaillaient alors au laboratoire d'acoustique du MIT. Jakobson et Halle publieront par la suite d'autres travaux ensemble, notamment l'ouvrage Fundamentals of Language (Jakobson \& Halle 1956). Halle a été l'élève de Jakobson à Columbia, avant d'enseigner au MIT, où il fera venir Chomsky en 1955. En 1968, il publie avec lui l'ouvrage The Sound Pattern of English (Chomsky \& Halle 1968). 
avez eu l'amabilité de m'envoyer. C'est une excellente description des procédés qui peuvent servir à identifier et à classifier les traits distinctifs de la parole considérée comme ensemble d'articulations et de perceptions. On aperçoit d'importants développements possibles de l'analyse en liaison avec le perfectionnement des appareils du type «Visible Speech $»^{74}$.

Je ne crois pas qu'on puisse faire à la méthode suggérée par B. Bloch ${ }^{75}$ cette concession de la déclarer «onerous but feasible» (p. 11). À mon avis cette procédure est entièrement « unfeasible » dans les termes où son auteur l'a proposée, en vertu des présuppositions nécessaires à l'identification des phonèmes.

Le titre de ce rapport (Speech Analysis) me dispense de souligner que l'ensemble de cette analyse, dans la mesure même où elle se précise et s'affine, tend à devenir l'auxiliaire de la psychologie plutôt que de la linguistique et à décrire l'homme plutôt que le système immatériel de la langue. Ce n'est pas une objection, mais seulement une caractérisation de cette description dont je suis le premier à reconnaître la valeur et l'intérêt.

Avec mes meilleurs remerciements et mes sentiments sincères,

E. Benveniste

ÉMILE BENVENISTE À ROMAN JAKOBSON

$10 / 06 / 1953$

Roman Jakobson Papers, MC 72, box 40, folder 4

Paris le 10 juin 1953

Cher ami,

Je vous remercie vivement de l'envoi de vos deux études. J'apprécie particulièrement l'importance de votre analyse sur la métrique comparée des langues slaves ${ }^{76}$. Vous avez, il me semble, mis hors de doute que ces schèmes métriques et les formes littéraires qu'ils caractérisent sont hérités et qu'ils attestent des types du slave commun ${ }^{77}$. Très remarquable aussi, pour la méthode, est la relation que vous établissez entre la structure phon $\bullet<\mathrm{i}>$ que et la structure métrique de la

74 Le Bell's visible speech était un alphabet « universel » fait de diagrammes simplifiés décrivant la position des organes lors de l'articulation. On le doit à Alex Melville Bell (1867). Les travaux de phonétique acoustique de Potter, Kopp \& Green (1947) et de Joos (1948) y font référence.

75 Bernard Bloch (1907-1965). Dans les Preliminaries, la méthode d'analyse des sons du langage par Bloch est décrite comme « to decipher the phonemic pattern of a language from a mere examination of a sufficient number of recorded utterances » (voir Jakobson, Fant \& Halle 1963 [1952] : 11 et Bloch 1948: 3-46, auquel il est fait référence dans le texte).

76 Jakobson, Roman. 1952. Studies in Comparative Slavic Metrics, Oxford Slavonic Papers 3 : 21-66 republié en 1966 sous un nouveau titre: Slavic Epic Verse. Studies in Comparative Metrics. Selected Writings, vol. IV: Slavic Epic Studies. La Haye : Mouton \& Co. 414-463.

77 Dans ce long article, Jakobson tente de reconstruire à partir de divers mètres slaves (décasyllabe épique serbo-croate, vers tchèques, ukrainiens, biélorusses, russes...) un vers slave commun à ces différentes traditions épiques. 
langue. Meillet aurait été heureux de lire cette belle démonstration ${ }^{78}$.

J'ai lu aussi avec un vif intérêt l'article tricéphale de Language ${ }^{79}$. Mais ici les problèmes demanderaient une discussion qui ne peut guère se faire par écrit. Ma seule objection porterait plutôt sur la « logique » de la représentation ainsi dégagée. Il ne me semble pas que la théorie de l'information permette de dessiner la configuration logique de la langue. Il nous faudra bien distinguer les problèmes du discours de ceux de la langue. Mais l'article donne beaucoup à réfléchir.

Votre amicalement dévoué

\section{E. Benveniste}

\section{[15] \\ ROMAN JAKOBSON À ÉMILE BENVENISTE \\ $01 / 09 / 1954$ \\ Roman Jakobson Papers, MC 72, box 40, folder 4}

Professor E. Benveniste

1 rue Monticelli

Paris 14, France

September 1,1954

Cher ami,

May I bring to your attention our very brilliant and promising student in linguistics, Mr. Calvert Watkins ${ }^{80}$, who will study in Paris in the coming academic year and who is a great admirer of your linguistic work.

I have not felt very well this summer and am recovering out of town. As soon as I feel better I hope to write to you about a few problems of common interest.

78 L'article de Jakobson se clôt sur une section intitulée « The Slavic Contribution to Comparative IndoEuropean Metrics » qui s'inscrit dans le prolongement des travaux de métrique comparée d'Antoine Meillet. Dans Les Origines indo-européennes des mètres grecs (1923), celui-ci avait démontré que les similitudes observables entre des mètres grecs et védiques étaient imputables à une structure métrique commune au stade indo-européen. L'ouvrage, fondateur pour la métrique comparée, posait la question de l'élargissement de la comparaison aux vers des langues baltiques et slaves. C'est cet élargissement que propose Jakobson dans son article. Il cite une lettre de Meillet reçue en décembre 1929 - « Je suis bien heureux de voir que vous apercevez le moyen de relier le mètre de la chanson baltique et slave aux mètres indo-européens. J'avais bien l'instinct que la question se pose. Mais, faute de travaux préparatoires, je n'ai pu y toucher »-et il conclut : "The testimony of the third witness to the foundations of the Indo-European verse may now join those of Greek and Vedic. » Un second élargissement intervient une dizaine d'années plus tard avec les travaux de Calvert Watkins (1963) qui intègrent à la comparaison un quatrième témoin : les vers irlandais. Sur l'histoire de la métrique comparée et le rôle qu'y joue l'article de Jakobson de 1952, voir Bader (1988), Watkins (1995 : 12 seq.), West (2007 : 45 seq.).

79 Jakobson, Roman, E. Colin Cherry \& Morris Halle. 1953. Toward the Logical Description of Languages in their Phonemic Aspect. Language 29(1) : 34-46.

80 Calvert Watkins (1933-2013) est l'étudiant de Jakobson à Harvard de 1954 à 1959 avant de devenir son collègue. En 1954-1955, il étudie à l'École pratique des hautes études auprès de Benveniste. Poursuivant la voie de Jakobson dans le domaine de la métrique et de la poétique comparées, Watkins revendique également l'empreinte de Benveniste dans ses travaux. Watkins a contribué à la reconnaissance du travail de Benveniste aux États-Unis dans le domaine des études indo-européennes. 
With best wishes,

Sincerely yours,

Roman Jakobson

\author{
[16] \\ ROMAN JAKOBSON À EMILE BENVENISTE \\ 23/02/1955 \\ Roman Jakobson Papers, MC 72, box 40, folder 4
}

February 23, 1955

Dear Benveniste:

Finally I have returned to my university work and hope also to be able soon to complete my book on sound and meaning ${ }^{81}$. I was very happy to have and to read your paper from Journal de Psychologie ${ }^{82}$, which gives such a penetrating insight into the focal problems of contemporary linguistics and which proved to me once again how close our ways in the science of language are. I would be very happy to resume our personal discussions and I hope to see you in the near future either on this or on your side of the Atlantic. Two of my papers on the utility of aphasia for general linguistics are in press ${ }^{83}$ and will be sent to you as soon as they appear. I am eager to learn your criticism.

With best wishes

Yours sincerely,

Roman Jakobson

ÉMILE BENVENISTE À ROMAN JAKOBSON

$10 / 10 / 1956$

Roman Jakobson Papers, MC 72, box 40, folder 4

1 rue Monticelli

Paris $14^{\mathrm{e}}$

Le 10 oct. 56

Cher ami,

Puisque vous avez reçu le beau volume que vos amis vous ont dédié ${ }^{84}$, je veux

81 Sur ce projet d'ouvrage, voir les notes de la lettre du 17/03/1948.

82 Il s'agit de «Tendances récentes en linguistique générale » (Benveniste 1954).

83 Il s'agit probablement, d'une part, de « Aphasia as a linguistic problem » (Jakobson 1955) et, d'autre part, de la seconde partie de l'ouvrage Fundamentals of Language (Jakobson \& Halle 1956: 53-82) intitulée « Two Aspects of Language and Two Types of Aphasic Disturbances » qui est signée de Jakobson seul, et qui reprend en partie le premier texte.

84 Il s'agit du volume d'hommage intitulé For Roman Jakobson. Essays on the Occasion of his Sixtieth Birthday, 11 October 1956 (Halle, Lunt \& McLean 1956). Benveniste contribue à ce volume par son texte « La nature des pronoms » (Benveniste 1966 : 251-257). Parmi les contributeurs, on trouve également LéviStrauss et Chomsky. 
y ajouter mes plus sincères félicitations et mes vœux affectueux. Que cet anniversaire vous apporte joie et fierté !

Votre

E. Benveniste

\section{[18] \\ ROMAN JAKOBSON À ÉMILE BENVENISTE 20/05/1966 \\ Roman Jakobson Papers, MC 72, box 40, folder 4}

Professor E. Benveniste

École Pratique des Hautes Études

1 , rue Monticelli,

Paris XIV, France

May 20, 1966

Dear Friend,

Warmest thanks and best congratulations on your splendid volume ${ }^{85}$. It is very important to have your papers on general linguistics brought together. My students were happy to learn this news. For me it was a great pleasure to reread your succinct studies which I already knew and those which were published in nonlinguistic periodicals and which had escaped my attention. In particular your illuminating discussion of the Oxford philosophical classification of verbs ${ }^{86}$ was immediately used by me for my grammatical analysis of a Dante sonnet ${ }^{87}$. As soon as it appears you will, naturally, have a reprint. I hope that you received the Epic volume of my Selected Writings ${ }^{88}$ which I asked Mouton to send you directly from The Hague. Two further volumes are in press ${ }^{89}$.

I was very sorry about being unable, because of urgent commitments, to attend the Philadelphia Symposium ${ }^{90}$ and to have missed the opportunity to welcome you in this country. I just learned, however, that I have to be in Paris for several days in the middle of March, 1967, and I hope very much to have a good chat with

85 Problèmes de linguistique générale (Benveniste 1966).

86 « La philosophie analytique et le langage » (Benveniste $1966: 267-276)$.

87 « Vocabulorum constructio in Dante's sonnet "Se vedi li occhi miei” » (Jakobson \& Valesio 1966: 7

33). L'article cite effectivement Benveniste et sa définition des énoncés performatifs contre la position d'Austin.

88 Slavic Epic Studies est le quatrième volume des Selected Writings de Jakobson (Jakobson 1966).

89 Il s'agit probablement du volume 2, Word and Language (1971) et du volume 3, Grammar of Poetry and Poetry of Grammar que Jakobson mentionne dans ses lettres à Lévi-Strauss de la même période (Jakobson \& Lévi-Strauss 2018 : 278-281). Ce volume ne paraîtra finalement qu'en 1981, enrichi par les nombreuses microscopies de poèmes que Jakobson développe dans les années soixante-dix et par ses réponses aux critiques que suscite son approche poétique, en particulier celles de Riffaterre, de Mounin et de Culler, auxquelles le « retrospect » de ce volume répond.

90 La Third Indo-European Conference se tient à l’Université de Philadelphie en avril 1966. Benveniste contribue aux actes de ce colloque par un texte intitulé « Les valeurs économiques dans le vocabulaire indoeuropéen »(Benveniste $1970: 307-320)$. 
you then. According to what I heard from Kurylowicz ${ }^{91}$, our views on the current problems of linguistic life on both hemispheres continue to be convergent.

With best wishes,

Yours devotedly,

Roman Jakobson

[19]

ÉMILE BENVENISTE À ROMAN JAKOBSON

25/06/1966

Roman Jakobson Papers, MC 72, box 138

\author{
à Roman Jakobson \\ pour renouveler en 1966
}

le témoignage que je lui ai rendu en 1956 ;

pour ajouter ma contribution

à un problème qui a suscité ses propres recherches ${ }^{92}$;

pour l'assurer de mes vœux les plus amicaux

au seuil de cette nouvelle décennie d'âge et de travail.

Paris, le 25 juin 1966

E. Benveniste

[20]

ÉMILE BENVENISTE À ROMAN JAKOBSON

15/07/1967

Roman Jakobson Papers, MC 72, box 40, folder 4

1 rue Monticelli

Paris $14^{\mathrm{e}}$

Le $15 / 7 / 67$

Mon cher ami,

Encore une belle étude de votre main experte que cette analyse de la « vocabulorum constructio » dans un sonnet de Dante ${ }^{93}$. Je suis, moi aussi, en ce moment occupé par les problèmes de la langue poétique mais à un point de vue un peu différent, celui de la sémiologie ; cela me paraît si difficile que je ne suis pas

91 Jerzy Kuryłowicz (1895-1978) est un indo-européaniste majeur du XX $X^{\mathrm{e}}$ siècle. De 1923 à 1925, il est l'étudiant de Meillet à l'École pratique des hautes études où il côtoie Benveniste. Professeur de linguistique générale à l'Université Jagellon de Cracovie depuis 1948, Kuryłowicz est, de 1964 à 1966, professeur invité à Harvard. Il est également présent à la Third Indo-European Conference de Philadelphie avec une contribution sur « The Quantitative Meter of Indo-European ».

92 «Les relations lexicales slavo-iraniennes», publié dans le volume To Honor Roman Jakobson: Essays on the Occasion of his 70. Birthday, 11 October 1966 (Benveniste 1967 : 197-202).

93 Voir Jakobson \& Valesio 1966, déjà mentionné plus haut. 
certain d'aboutir à rien de publiable ${ }^{94}$. Nous en reparlerons bientôt.

Toutes mes amitiés

E. Benveniste

\author{
[21] \\ ÉMILE BENVENISTE À ROMAN JAKOBSON \\ $16 / 05 / 1968$ \\ Roman Jakobson Papers, MC 72, box 40, folder 4
}

1 , rue Monticelli

Paris, le 16 mai 1968

Paris $14^{\mathrm{e}}$

Cher ami,

Merci pour votre lettre relative au Congrès de Varsovie ${ }^{95}$. Je suis heureux d'apprendre qu'il aura lieu malgré les changements survenus ces temps derniers.

Mais aucun message de Varsovie ne m'est parvenu depuis que St. Żołkiewski a été démis ${ }^{96}$. Je ne sais pas qui l'a remplacé ni quelles sont les dispositions prises par le Comité d'organisation, etc. En dernier lieu, Żołkiewski m'avait demandé quelques renseignements en vue du visa, et je n'ai pu lui répondre, car sa lettre précédait de peu la mesure officielle qui a été prise contre lui.

Je souhaite donc que le responsable actuel se fasse connaître de moi et me dise où en sont les choses.

Vous savez peut-être que toute l'Université traverse ici une crise profonde, dans l'exaltation et la confusion ${ }^{97}$. C'est une expérience passionnante, dont on ne peut encore prévoir l'issue.

Votre amicalement dévoué,

\title{
E. Benveniste
}

94 Il s'agit de la recherche, restée manuscrite, sur «la langue de Baudelaire» (voir Benveniste 2011 ; Laplantine 2011 ; Adam \& Laplantine 2012).

95 Symposium international de sémiotique, Varsovie, 25 août- $-^{\text {er }}$ septembre 1968. Benveniste y fait une communication sur « La distinction entre le sémiotique et le sémantique » dont il reprend la matière pour son article «Sémiologie de la langue» (Benveniste 1969). Une communication de Jakobson (« Toward a Classification of Semiotic Systems ») figure au programme du symposium, mais il ne s'y rend pas en raison de la situation politique dans le bloc de l'Est qui culmine avec la répression du printemps de Prague. Les actes sont publiés sous la direction de Josette Rey-Debove, sous le titre Recherches sur les systèmes signifiants. À l'issue de ce symposium est créée l'Association internationale de sémiotique (International Association for Semiotic Studies) dont Benveniste est le premier président. Après l'attaque cérébrale dont Benveniste est victime en décembre 1969, Jakobson accepte d'en assurer la présidence jusqu'en 1971 où lui succède Cesare Segre.

96 Stefan Żółkiewski (1911-1991) est un théoricien et historien de la littérature polonais. Ancien ministre de l'Enseignement supérieur et membre du comité central du parti, il est alors secrétaire de la section des sciences sociales de l'Académie des sciences polonaises. Sa révocation de l'université en mars 1968 prend place dans le cadre de la répression qui suit le mouvement étudiant et dans la purge antisémite menée par le gouvernement polonais au sein du parti.

97 Benveniste fait référence au mouvement social de mai 68 : la Sorbonne est occupée depuis le 3 mai ; le 13 mai commencent la grève générale et les occupations d'usines. L'intérêt que Benveniste porte à cette contestation confirme ce que l'on sait sur son orientation politique, « très à gauche » comme l'écrit Milner (2008 [2002] : 139). 


\section{BIBLIOGRAPHIE}

\section{Sources archivistiques}

Archives de la Fondation Rockefeller. RF. RG 1.2. Series 500R. Box 12. Folder 113.

Massachusetts Institute of Technology, Institute Archives and Special Collections, Roman Jakobson Papers, MC 72.

\section{Autres sources}

Adam, Jean-Michel \& Chloé Laplantine, éd. 2012. Semen 33. Les notes manuscrites de Benveniste sur la langue de Baudelaire [https://journals.openedition.org/ semen/9442, consulté le 20/09/2021].

Annuaire du Collège de France. 1947-1948 et 1948-1949. Paris: Imprimerie nationale.

Bader, Françoise. 1988. Meillet et la poésie indo-européenne. Cahiers Ferdinand de Saussure 42 : 97-125.

Bader, Françoise. 2012. Lettres d'Émile Benveniste à Claude Lévi-Strauss. Contribution à la biographie d'Émile Benveniste. Per Roberto Gusmani. 1: Linguaggi, culture, letterature. 2: Linguistica storica e teorica. Studi in ricordo, dir. par Giampaolo Borghello e Vincenzo Orioles. Udine : Forum. 227-249 [http://forum editrice.it/percorsi/lingua-e-letteratura/studi-in-onore/per-roberto-gusmani/lettres -d2019emile-benveniste-a-claude-levi, consulté le 20/09/2021].

Bank Jensen, Viggo \& Giuseppe D'Ottavi. 2020. From the Early Years of Phonology. The Roman Jakobson-Eli Fischer-Jorgensen Correspondence (1949-1982). Copenhague : The Royal Danish Academy of Sciences and Letters.

Bell, Alexander Melville. 1867. Visible Speech: The Science of Universal Alphabetics; or Self-Interpreting Physiological Letters, for the Writing of All Languages in One Alphabet. London : Simpkin, Marshall \& Co.

Benveniste, Émile. 1939. Répartition des consonnes et phonologie du mot. Travaux $d u$ Cercle linguistique de Prague 8 : 3-11.

Benveniste, Émile. 1948. Noms d'agent et noms d'action en indo-européen. Paris : Adrien-Maisonneuve.

Benveniste, Émile. 1954. Tendances récentes en linguistique générale. Journal de psychologie normale et pathologique 74(1-2) : 130-145.

Benveniste, Émile. 1966. Problèmes de linguistique générale 1. Paris : Gallimard.

Benveniste, Émile. 1967. Les relations lexicales slavo-iraniennes. To Honor Roman Jakobson: Essays on the Occasion of his Seventieth Birthday. 11 October 1966. La Haye \& Paris : Mouton. 197-202.

Benveniste, Émile. 1969. Sémiologie de la langue. Semiotica $1: 1-12$ et $2: 127-135$.

Benveniste, Émile. 1970. Les valeurs économiques dans le vocabulaire indoeuropéen. Indo-European and Indo-Europeans. Papers Presented at the Third Indo-European Conference at the University of Pennsylvania [21-23 avril 1966], éd. par G. Cardona, H. M. Hoenigswald \& A. Senn. Philadelphie : University of Pennsylvania Press. 307-320. 
Benveniste, Émile. 2011. Baudelaire. Présentation et transcription de Chloé Laplantine. Limoges : Lambert-Lucas.

Benveniste, Émile. 2012. Dernières leçons. Collège de France, 1968 et 1969, éd. par Jean-Claude Coquet et Irène Fenoglio. Paris : EHESS, Seuil \& Gallimard.

Bloch, Bernard. 1948. A Set of Postulates for Phonemic Analysis. Language 24(1) : 3-46.

Bossuat, Robert. 1948. La geste du prince Igor, épopée russe du XII siècle [compte rendu]. Bibliothèque de l'École des Chartes 107(1) : 123-124.

Buswell, James O., III. 1950. Some Comments on Hockett's "Language 'and' Culture: A Protest”. American Anthropologist 52(2) : 284-286.

Chevalier, Jean-Claude. 1997. Trubetzkoy, Jakobson et la France, 1919-1939. Cahiers de l'ILSL 9 : 31-43.

Chomsky, Noam \& Morris Halle. 1968. The Sound Pattern of English. New York : Harper \& Row.

Coste, Daniel. 2006. Français élémentaire, débats publics et représentations de la langue. Documents pour l'histoire du français langue étrangère ou seconde 36 [http://journals.openedition.org/dhfles/1181, consulté le 20/09/2021].

Dixon, R. M. W. 2007. Roman Jakobson and the Two-Dollar Bills. Historiographia Linguistica 34(2/3) : 435-440.

Grégoire, Henri, Roman Jakobson \& Marc Szeftel, éd. 1948. La geste du prince Igor, épopée russe du XII siècle. New York : Rausen Brothers (Annuaire de l'Institut de philologie et d'histoire orientales et slaves, 8).

Halle, Morris, Horace G. Lunt \& Hugh McLean, éd. 1956. For Roman Jakobson. Essays on the Occasion of his Sixtieth Birthday, 11 October 1956. La Haye : Mouton \& Co.

Havránková, Marie \& Vladimir Petkevič. 2014. Pražská škola v korespondenci. Dopisy z let 1924-1989. Prague : Karolinum.

Hjelmslev, Louis \& Eli Fischer-Jorgensen. 1950. Sixième Congrès international des linguistes. Paris 1948. Acta Linguistica 5(1) : 56-60.

Hockett, Charles F. 1950. Language "and" Culture: A Protest. American Anthropologist 52(1) : 113.

Jakobson, Roman. 1941. Kindersprache, Aphasie und allgemeine Lautgesetze. Uppsala : Almqvist \& Wiksells.

Jakobson, Roman. 1949a. The Phonemic and Grammatical Aspects of Language in their Interrelations. Actes du Sixième Congrès international des linguistes, éd. par Michel Lejeune. Paris : Klincksieck. 5-18 et 601.

Jakobson, Roman. 1949b. On the Identification of Phonemic Entities. Travaux du Cercle linguistique de Copenhague 5 : 205-213.

Jakobson, Roman. 1950. Slavic mythology. Funk \& Wagnalls Standard Dictionary of Folklore, Mythology, and Legend. New York : Funk \& Wagnalls. 1025-1028.

Jakobson, Roman. 1952. Slavic Epic Verse. Studies in Comparative Metrics. Selected Writings, vol. IV: Slavic Epic Studies. La Haye : Mouton \& Co. 416-463.

Jakobson, Roman. 1955. Aphasia as a Linguistic Problem. On Expressive Language, éd. par H. Werner. Worcester : Clark University Press. 69-81. 
Jakobson, Roman. 1957. Shifters, verbal Categories, and the Russian verb. Cambridge, Mass : Harvard University.

Jakobson, Roman. 1966. Selected writings, vol. IV: Slavic Epic Studies. La Haye : Mouton \& Co.

Jakobson, Roman. 1975a. Les règles des débats grammaticaux. Langue, discours, société. Pour Émile Benveniste, éd. par Julia Kristeva, Jean-Claude Milner \& Nicolas Ruwet. Paris : Seuil. 11-25.

Jakobson, Roman. 1975b. Glosses on the Medieval Insight into the Science of Language. Mélanges linguistiques offerts à Émile Benveniste. Paris : Société linguistique de Paris \& Peeters. 289-303.

Jakobson, Roman. 1979. Coup d'œil sur le développement de la sémiotique. Panorama sémiotique. Actes du Premier Congrès de l'Association internationale de sémiotique, Milan, juin 1974, éd. par Seymour B. Chatman, Umberto Eco et Jean-Marie Klinkenberg. La Haye : Mouton. 3-18.

Jakobson, Roman. 1980. Retrospect. Selected Writings, vol. III. La Haye : Mouton. 765-789.

Jakobson, Roman. 2014 [1937]. Prof. Benveniste v Brně / Prof. Benveniste in Brno, Selected Writings, vol. IX(2). La Haye : De Gruyter Mouton. 246-247.

Jakobson, Roman, E. Colin Cherry \& Morris Halle. 1953. Toward the Logical Description of Languages in their Phonemic Aspect. Language 29(1) : 34-46.

Jakobson, Roman, Gunnar Fant \& Morris Halle. 1963 [1952]. Preliminaries to Speech Analysis: the Distinctive Features and their Correlates. Cambridge : The MIT Press [https://hdl.handle.net/2027/heb.08432, consulté le 21/09/2021].

Jakobson, Roman \& Morris Halle. 1956. Fundamentals of Language. La Haye : Mouton \& Co.

Jakobson, Roman, Claude Lévi-Strauss, C. F. Voegelin \& Thomas A. Sebeok. 1953. Results of the Conference of Anthropologists and Linguists. Baltimore : Waverly Press (Memoirs of the International Journal of American Linguistics, 8).

Jakobson, Roman \& Claude Lévi-Strauss. 1962. « Les Chats » de Charles Baudelaire. L'Homme 2(1) : 5-21.

Jakobson, Roman \& Claude Lévi-Strauss. 2018. Correspondance 1942-1982. Préfacé, édité et annoté par Emmanuelle Loyer et Patrice Maniglier. Paris : Seuil.

Jakobson, Roman \& John Lotz. 1941. Axiomatik eines Verssystems am mordwinischen Volkslied dargelegt (Thesen zu einem Vortrag im Ungarischen Institut am achten April 1941). Stockholm : Ungerska Institutet.

Jakobson, Roman \& John Lotz. 1949. Notes on the French Phonemic Pattern. Word 5(2) : 151-158.

Jakobson, Roman \& John Lotz. 1968. Két tanulmány, trad. par S. J. Petőfi et G. Szépe. Budapest : Magyar Nyelvtudományi Társaság 119. szám kiadvanyai.

Jakobson, Roman, Gladys A. Reichard \& Elizabeth Werth. 1949. Language and Synesthesia. Word 5(2) : 224-233.

Jakobson, Roman \& Paolo Valesio. 1966. Vocabulorum constructio in Dante's sonnet « Se vedi li occhi miei ». Studi danteschi 43 : 7-33. 
Joos, Martin. 1948. Acoustic Phonetics. Baltimore : Linguistic Society of America (Language Monograph, 23).

Joseph, John E., Chloé Laplantine \& Georges-Jean Pinault. 2020. Lettres d'Émile Benveniste à Claude Lévi-Strauss (1948-1967). Histoire Épistémologie Langage 42(1) : $155-181$.

Kobližek, Tomáš \& Eva Krásová. 2019. Émile Benveniste et le Cercle linguistique de Prague. Émile Benveniste, 50 ans après les "Problèmes de linguistique générale », éd. par G. D’Ottavi \& I. Fenoglio. Paris : Éditions Rue d'Ulm. 163-193.

Laplantine, Chloé. 2011. Émile Benveniste, l'inconscient et le poème. Limoges : Lambert-Lucas.

Lejeune, Michel, éd. 1949. Actes du Sixième Congrès international des linguistes [Paris, 19-24 juillet 1948]. Paris : Klincksieck.

Linguistic Society of America. 1951. Proceedings of the Linguistic Society of America, at the Twelfth Summer Meeting, Ann Arbor, Michigan, July 28-29, 1950. Bulletin 24 :3-4 [https://www.jstor.org/stable/43673053, consulté le 28/04/2021].

Loyer, Emmanuelle. 2015. Lévi-Strauss. Paris : Flammarion.

MacRae Taylor, Douglas. 1950. Language "and" or "in" Culture. American Anthropologist 52(4) : 559-560.

Martinet, André. 1994. An Early History of Word. Vol. 1 to 25. Word 45(1) : 27-37.

Mazon, André. 1940. Le Slovo d'Igor. Paris : Droz.

Milner, Jean-Claude. 2008 [2002]. Le Périple structural. Figures et paradigmes. Paris : Verdier.

Potter, Ralph K., Georg A. Kopp \& Harriet C. Green. 1947. Visible speech. New York : D. Van Nostrand.

Puech, Christian. 1998. Manuélisation et disciplinarisation des savoirs de la langue. Les Carnets du Cediscor 5 : 15-30 [https://doi.org/10.4000/cediscor.267, consulté le 20/09/2021].

Reichard, Gladys A. 1950. Language and Cultural Pattern. American Anthropologist 52(2) : 194-204.

Ronat, Mitsou. 1972. Jakobson au Collège de France. Les lettres françaises 1426 [814 mars 1972] : 3-4.

Roudet, Robert. 2011. Mazon et le Slovo d'Igor. Revue des études slaves 82(1) : 5567.

Sebeok, Thomas A. 1950. Reply to Hockett. American Anthropologist 52(2) : 286.

Silva-Fuenzalida, Ismael. 1949. Ethnolinguistics and the Study of Culture. American Anthropologist 51(3) : 446-456.

Tesnière, Louis. 1948. La geste du prince Igor, épopée russe du XII siècle [compte rendu]. Bulletin de la Société de linguistique de Paris 44(2) : 144-148.

Testenoire, Pierre-Yves. 2019. Compléments à la correspondance Jakobson - LéviStrauss. Acta Structuralica 4 [https://doi.org/10.19079/actas.2019.4.2, consulté le 21/09/2021].

Testenoire, Pierre-Yves. À paraître. Jakobson \& Co. La linguistique à l'École libre des hautes études. La linguistique et ses formes historiques d'organisation et de production, éd. par Didier Samain \& Pierre-Yves Testenoire. Paris : HEL Livres. 
Toutain, Anne-Gaëlle. 2014. Les embrayeurs : de la langue au langage. Bulletin de la Société de linguistique de Paris 109(1) : 155-199.

Troubetzkoy, Nikolaï S. 2006, Correspondance avec Roman Jakobson et autres écrits, éd. par Patrick Sériot. Lausanne : Payot.

Voegelin, Charles F. 1949. Linguistics without Meaning and Culture without Words. Word 5(1) : 36-45.

Voegelin, Charles F. 1950. A "Testing Frame” for Language and Culture. American Anthropologist 52(3) : 432-435.

Watkins, Calvert. 1963. Indo-European Metrics and Archaic Irish Verse. Celtica 6 : 194-249.

Watkins, Calvert. 1983. Roman Jakobson and Slavic Mythology. A Tribute to Roman Jakobson 1896-1982. Berlin, New York \& Amsterdam : De Gruyter. 39-46.

Watkins, Calvert. 1995. How to Kill a Dragon: Aspects of Indo-European Poetics. New York \& Oxford : Oxford University Press.

West, Martin Litchfield. 2007. Indo-European Poetry and Myth. New York : Oxford University Press. 